\title{
Peripheral Artery Disease on The Prognosis Value of Patients with Stable Coronary Artery Disease Undergoing Percutaneous Coronary Intervention: A Retrospective, Single-Center Cohort Study
}

\author{
Zeyi Cheng, ${ }^{1 *}$ Miaomiao Qi, ${ }^{2 *}$ Zekun Lang, ${ }^{3}$ Tingting Fang, ${ }^{4}$ Mahboob Alam ${ }^{5}$ Jing Yu, ${ }^{2}$ Yingqiang Guo ${ }^{1}$ \\ ${ }^{1}$ Department of Cardiovascular Surgery, West China Hospital, Sichuan University, No. 37, Guoxue Alley, Chengdu, Sichuan 610041, China; \\ ${ }^{2}$ Department of Cardiology, The Second Hospital of Lanzhou University, Lanzhou, Gansu Province, 730000, China; \\ ${ }^{3}$ The Medical School of Lanzhou University, Lanzhou, Gansu Province, 730000, China; \\ ${ }^{4}$ Department of Cardiology, West China Hospital, Sichuan University, No. 37, Guoxue Alley, Chengdu, Sichuan 610041, China \\ ${ }^{5}$ Department of Medicine, Division of Cardiovascular Medicine, Baylor College of Medicine, Houston, Texas, USA.
}

\section{ABSTRACT}

Objective: The purpose of this investigation aimed to clarify the impact of peripheral artery disease (PAD) on the prognosis value of patients with stable coronary artery disease (CAD) who underwent percutaneous coronary intervention (PCI).

Methods: The SPSS 16 software was used for secondary analysis of DRYAD database data. A total of 204 patients were enrolled from Shinonoi General Hospital for newly diagnosed stable CAD and received PCI performance between October 2014 and October 2017. Patients with old myocardial infarction (MI) were excluded. We divided patients into two groups with PAD and without PAD. The primary endpoints were major adverse cardiac events (MACE, defined as all-cause death, non-fatal MI, and non-fatal stroke) and cardiovascular events (defined as cardiovascular death, non-fatal MI, and non-fatal stroke). The secondary outcomes were the individual components of the composite primary outcomes. The median follow-up time was 783 days.

Results: No statistical difference was found between PAD and non-PAD patients of lesional characteristics. Spearman's rank correlations indicate diabetes mellitus $(\mathrm{DM})(\mathrm{P}=0.019)$ and $\mathrm{HbAlc}(\mathrm{P}=0.009)$ are positively correlated with $\mathrm{PAD}$. In Kaplan-Meier analysis, patients with $\mathrm{PAD}$ predicted poor prognosis in MACE $(\mathrm{P}<0.05)$ and cardiovascular events $(\mathrm{P}<0.05)$. In Multivariable Cox proportional hazards analysis, patients with PAD independently predicted MACE and cardiovascular events.

Conclusions: PAD is a significant mediator for the prognosis of patients with stable CAD who underwent PCI treatment.

Received August 7, 2021; accepted August 30, 2021.

*Zeyi Cheng and Miaomiao Qi contributed equally.

Correspondence: Mabboob Alam, Department of Medicine, Division of Cardiovascular Medicine, Baylor College of Medicine, Houston, Texas, USA. (e-mail: malam@bcm.edu); Yingqiang Guo, Department of Cardiovascular Surgery, West China Hospital, Sichuan University, No. 37, Guoxue Alley, Chengdu, Sichuan 610041, China (e-mail: drguoyq@hotmail.com).

\section{INTRODUCTION}

PAD is defined as a vascular disease mainly affecting lower extremities characterized by atherosclerotic vascular [Abola 2020]. The prevalence of PAD showed an increasing trend worldwide [Fowkes 2013]. In Europe, the prevalence of PAD reached 5.3\% [Olinic 2018]. Its clinical manifestations vary from the reduction of Ankle-Brachial index (ABI) without symptoms, intermittent claudication (IC) to severe ischemic symptoms [Norgren 2007]. Patients with PAD have higher risks of stroke, myocardial infarction (MI) and even cardiovascular mortality [Morris 2014].

$\mathrm{CAD}$ is the main cause of death in many countries. In Europe, CAD is responsible for nearly $20 \%$ of deaths caused by cardiovascular diseases [Roth 2017]. Because atherosclerosis is a systemic condition, CAD and PAD present the common pathogenesis and risk factors for development, such as smoking, dyslipidemia, hypertension, and diabetes mellitus [Bhatt 2006]. Several studies have suggested that the incidence of major cardiovascular events among patients with symptomatic $\mathrm{PAD}$ is higher than those with symptoms of CAD [McKenna 1991]. However, the prevalence of patients with both CAD and PAD ranges from 54\% to 69\% [Ryu 2012; Nishijima 2017; Global 2017]. These patients developed a particularly poor long-term prognosis. PCI is widely used to improve the survival and prognosis of CAD. The purpose of this investigation aimed to find the impact of PAD on the prognosis value of patients with stable CAD who underwent PCI.

\section{METHODS}

Data resource: The data used in this study comes from an open access database DRYAD website (https://DATADRYAD. org). The site allows users to download the original data for Dryad, Dataset (https://doi.org/10.5061/dryad.fn6730j).

Study design: The study design previously has been described [Suzuki 2019]. It was a retrospective, single-center cohort study. The study included patients admitted to Shinonoi General Hospital between October 2014 and October 2017 for newly diagnosed stable CAD who received PCI 
performance. Patients with old myocardial infarction (MI) were excluded. A total of 204 patients were enrolled in the study. We performed a post-hoc analysis of MACE and cardiovascular events, according to reported PAD status at baseline. Patients were divided into two groups: the with PAD group and without PAD group.

PAD was diagnosed by ischemic pain at rest, an ulcer, or gangrene in one or both legs attributed to the objectively proven arterial occlusive disease. Stable CAD was defined as angiographic stenosis $\geq 90 \%$ in the epicardial coronary artery or angiographic stenosis $\geq 75 \%$ in the epicardial coronary artery with either a symptom of chest pain induced by exercise or evidence of stress-induced ischemia via any clinical stresstesting modality. Old myocardial infarction was diagnosed by the cardiologists using all available data, including symptoms, laboratory findings, electrocardiograms, echocardiograms, and coronary angiograms. Coronary angiography and PCI were performed, according to the guidelines and standard protocols.

An investigator collected the information of the enrolled patients, including clinical characteristics, medical history, related risk factors, comorbidities, medications, and the data of all examinations. The investigation is consistent with the principles outlined in the Declaration of Helsinki. The study was approved by the Shinonoi General Hospital Ethics Committee, and written informed consent was obtained.

Endpoints: The primary outcomes were major adverse cardiac events (MACE; defined as all-cause death, non-fatal MI, and non-fatal stroke) and cardiovascular events, including cardiovascular death, non-fatal MI, and non-fatal stroke. The secondary outcomes were the individual components of the composite primary outcomes. The median follow-up time was 708 days.

Statistical analysis: Continuous variables are summarized as mean \pm standard deviation, if normally distributed, and as median [interquartile range], if non-normally distributed. Normality was assessed by the Shapiro-Wilk W-test. Comparisons of baseline characteristics were conducted with a contingency table. Pearson's $\chi^{2}$ test was used for categorical variables, the $\mathrm{t}$-test was used for normally distributed continuous variables, and the Wilcoxon or Mann-Whitney test was used for non-normally distributed continuous variables. Spearman's rank correlation method was used as a nonparametric measure of the association between Alb and clinical indices. Patients were then divided into 2 groups, according to with or without PAD. Kaplan-Meier survival plots were calculated from baseline to the time of MACE and compared using the log-rank test. Cox proportional-hazards analysis was used to evaluate the independent prognostic utility of the presence of PAD. The covariates used were age, sex, body mass index (BMI), hemoglobin, estimated glomerular filtration rate (eGFR), and CRP. Because the study included only a small number of patients, a power calculation was performed. A $P$-value of $<0.05$ was considered statistically significant. All statistical analyses were analyzed by SPSS Statistics.

\section{RESULTS}

Baseline characteristics: A total of 204 patients were enrolled (median age, 73 years old). The baseline characteristics are shown in Table 1. (Table 1) Of these, 53 patients (26\%) had the presence of PAD at baseline. Compared with CAD patients without PAD, CAD patients with PAD had higher Hb1c $(6.3 \%$ [5.8\%-7.0\%] vs. $6.0 \%$ [5.6\%-6.7\%], $P$ $=0.009)$, Triglycerides $(134 \%$ [87\%-199\%] vs. $106 \%$ [78\%$149 \%$ ], $P=0.024)$ and presence of diabetes mellitus $(26 \%$ vs. $47 \%, P=0.019)$. However, no significant correlations were detected between PAD and these clinical indices. (Table 2) The baseline lesional characteristics are shown in Table 3. (Table 3) Among the PAD patients, 28\% (15/53) had multivessel disease, and 7.5\% (4/53) had CTO lesions. No statistical difference was found between PAD and non-PAD patients of lesional characteristics.

Clinical outcomes by PAD status: In this study, during the follow up of 1500 days, $14 \%$ (28/204) of patients experienced MACE. The PAD group had 24.5\% (13/53) patients who developed MACE, whereas the no PAD group had only 9.9\% (15/151). Patients with PAD indicated a higher risk of MACE $(24.5 \%$ vs. $9.9 \%, P=0.008)$. In multivariate Cox proportional hazards analysis of $\mathrm{PAD}$ patients, after adjusting for age, CRP and TG, PAD could predict the risk of MACE. (Table 4) Kaplan-Meier analysis combined with PAD could independently predict MACE (all-cause death, MI, and stroke) $(P=0.015)$. (Figure 2$)$ In addition, $\mathrm{PAD}$ patients also could predict cardiac events (cardiac death, MI, and stroke) $(P=0.034)$. (Figure 4) However, in terms of all-cause death events or cardiac death events, respectively, PAD patients had no ability of prediction. (Figure 1) (Figure 3) (Figure 5)

\section{DISCUSSION}

The study evaluated the impact of $\mathrm{PAD}$ on the prognosis value of stable CAD patients following PCI. The main finding is the significant interaction between PAD at admission and the poor prognosis in patients hospitalized with newly diagnosed stable CAD undergoing PCI treatment. PAD could predict the higher risk of MACE and cardiovascular events. In our study, we observed that $\mathrm{CAD}$ patients with $\mathrm{PAD}$ are at higher risk for MACE and cardiac events. The composited endpoint of all-cause death, MI, and stroke occurred in $24.5 \%$ of patients with PAD and $9.9 \%$ of patients without PAD. Concomitant PAD increases the rate of all-cause death, $\mathrm{MI}$, and stroke. Our conclusions are consistent with previous studies [Nikolsky 2004; O'Connor 1999]. Similarly, a retrospective registry study by Nikolsky et al. observed that patients with symptomatic PAD had higher in-hospital complications, 1-year mortality and MI [Nikolsky 2004]. O'Connor et al. found that peripheral vascular disease was an important predictor of in-hospital outcome for PCI [O'Connor 1999].

In the study, $25 \%$ of the stable CAD patients combined with PAD at baseline. This rate appears higher than those previously reported [Singh 2004; Chiu 2003]. In our study, we mentioned that patients in the PAD group had a higher level of TG than the no PAD group at baseline. CAD patients with PAD often are recognized as having systemic AS conditions, receiving more intensive lipid-lowering therapies to improve 
Table 1. Baseline characteristics

\begin{tabular}{|c|c|c|c|c|}
\hline Age (years) & $73[66-80]$ & $73[68-80]$ & $73[65-80]$ & 0.58 \\
\hline BMI & $23.4[21.0-25.7]$ & $22.7[20.2-25.5]$ & $23.8[21.1-25.7]$ & 0.218 \\
\hline Systolic blood pressure $(\mathrm{mmHg})$ & 136 [123-147] & $136[120-146]$ & 138 [125-147] & 0.179 \\
\hline Hypertension, n (\%) & $151(74)$ & $36(24)$ & $115(76)$ & 0.24 \\
\hline Dyslipidemia, n (\%) & $104(51)$ & $34(33)$ & $70(67)$ & 0.026 \\
\hline Diabetes mellitus, $\mathrm{n}(\%)$ & $73(36)$ & $26(36)$ & $47(64)$ & 0.019 \\
\hline Atrial fibrillation, $\mathrm{n}(\%)$ & $26(13)$ & $6(23)$ & $20(77)$ & 0.718 \\
\hline OCl, n (\%) & $35(17)$ & $17(49)$ & $18(51)$ & 0.001 \\
\hline \multicolumn{5}{|l|}{ Medication } \\
\hline Aspirin, n (\%) & $202(99)$ & $52(26)$ & $150(74)$ & 0.436 \\
\hline Thienopiridines, $\mathrm{n}(\%)$ & $200(98)$ & $50(25)$ & $150(75)$ & 0.024 \\
\hline Warfarin, n (\%) & $5(2.4)$ & $1(20)$ & $4(80)$ & 0.758 \\
\hline DOAC, n (\%) & $21(10)$ & $3(14)$ & $18(86)$ & 0.197 \\
\hline Statin, n (\%) & $111(54)$ & $33(30)$ & $78(70)$ & 0.182 \\
\hline Ezetimibe, n (\%) & $3(1.5)$ & $1(33)$ & $2(67)$ & 0.77 \\
\hline PPI, n (\%) & $135(66)$ & $32(24)$ & $102(76)$ & 0.344 \\
\hline ACE-Is, n (\%) & $19(9)$ & $3(16)$ & $16(84)$ & 0.287 \\
\hline $\mathrm{Alb}(\mathrm{g} / \mathrm{dL})$ & $4.0[3.6-4.3]$ & $3.9[3.5-4.3]$ & $4.1[3.7-4.4]$ & 0.123 \\
\hline eGFR $(\mathrm{mL} / \mathrm{min} / 1.73 \mathrm{~m} 2)$ & $40[53-75]$ & 59 [39-73] & $65[56-76]$ & 0.081 \\
\hline $\mathrm{AST}(\mathrm{U} / \mathrm{L})$ & 23 [18-29] & 21 [18-27] & 23 [19-29] & 0.073 \\
\hline $\operatorname{ALT}(U / L)$ & $18[14-26]$ & $17[12-26]$ & $16[14-27]$ & 0.18 \\
\hline T-Chol (mg/dL) & 184 [168-208] & $187[168-212]$ & 183 [163-206] & 0.476 \\
\hline HDL-Chol (mg/dL) & 49 [41-57] & 48 [36-58] & 49 [41-57] & 0.394 \\
\hline LDL-Chol (mg/dL) & 109 [90-129] & 105 [89-128] & $109[91-130]$ & 0.303 \\
\hline Triglycerides (mg/dL) & $107[83-160]$ & 134 [87-199] & 106 [78-149] & 0.024 \\
\hline $\mathrm{CRP}(\mathrm{mg} / \mathrm{dL})$ & $0.12[0.04-0.34]$ & $0.15[0.06-0.41]$ & $0.11[0.04-0.32]$ & 0.4 \\
\hline $\mathrm{CRP} / \mathrm{Alb} \times 100$ & $2.9[1.1-8.9]$ & $3.6[0.9-9.9]$ & $2.5[0.9-8.4]$ & 0.51 \\
\hline $\mathrm{HbA1c}(\%)$ & $6.0[5.7-6.7]$ & $6.3[5.8-7.0]$ & $6.0[5.6-6.7]$ & 0.009 \\
\hline
\end{tabular}


the outcomes [Olin 2010]. Besides, to improve the prognosis, therapies such as antihypertensive therapy and antiplatelet therapy are recommended for PAD patients.

There are several limitations to our study. First, our study is a post-hoc analysis, and all the findings should be considered as hypothesis-generating. Second, the number of enrolled patients is not large enough. Also, the median age of patients was a little high. So, the enrolled patients couldn't represent the general CAD populations. Meanwhile, we didn't have objective measures to define the diagnosis of PAD. Third, CAD patients with PAD had a higher risk of major bleeding complications after PCI [Saw 2006]. Unfortunately, the study didn't report the adverse event as the endpoint.

\section{CONCLUSION}

Patients with PAD and CAD are under a larger atherosclerotic burden. Meanwhile, these patients often have evidence of polyvascular disease. And they display a high rate of adverse cardiovascular events, including all-cause death, CV death,

Table 2. Univariate Spearman's rank correlations between PAD and clinical indices

\begin{tabular}{lcc}
\hline Variable & Spearman's Rank & $P$-value \\
\hline Diabetes mellitus & 0.164 & 0.019 \\
Atrial fibrillation & -0.025 & 0.719 \\
Past smoker & 0.017 & 0.809 \\
T-Chol $(\mathrm{mg} / \mathrm{dL})$ & 0.055 & 0.476 \\
HDL-Chol $(\mathrm{mg} / \mathrm{dL})$ & -0.061 & 0.394 \\
LDL-Chol $(\mathrm{mg} / \mathrm{dL})$ & -0.074 & 0.303 \\
CRP $(\mathrm{mg} / \mathrm{dL})$ & 0.06 & 0.4 \\
$\mathrm{CRP} / \mathrm{Alb} \times 100$ & 0.046 & 0.51 \\
Alb $(\mathrm{g} / \mathrm{dL})$ & -0.108 & 0.123 \\
HbA1c $(\%)$ & 0.189 & 0.009
\end{tabular}

$\mathrm{MI}$, and stroke. PAD could be a potent mediator for the prognosis of CAD patients. Further research is needed to clarify how to discern the high-risk patients in time and perform intensive therapy to improve the prognosis of these patients.

\section{ACKNOWLEDGEMENT}

Funding: This study was supported by National Natural Science Foundation of China (No. 81970345).

\section{REFERENCES}

Abola MTB, Golledge J, Miyata T, et al. 2020. Asia-Pacific Consensus Statement on the Management of Peripheral Artery Disease: A Report from the Asian Pacific Society of Atherosclerosis and Vascular Disease Asia-Pacific Peripheral Artery Disease Consensus Statement Project Committee. J Atheroscler Thromb. 27(8):809-907.

Tab; e 4. Multivariable Cox proportional hazards analysis of PAD patients

\begin{tabular}{lcc}
\hline Variable & $\mathrm{HR}(95 \% \mathrm{Cl})$ & P-value \\
\hline PAD adjusted for & & \\
Age, Sex & $2.290(0.893-5.867)$ & 0.084 \\
Age, BMI & $2.186(0.849-5.625)$ & 0.105 \\
Age, Hb & $2.232(0.869-5.732)$ & 0.095 \\
Age, eGFR & $1.972(0.769-5.059)$ & 0.158 \\
Age, CRP & $2.799(1.063-7.369)$ & $0.037^{*}$ \\
Age, sBP & $2.263(0.887-5.775)$ & 0.088 \\
Age, dBP & $2.332(0.905-6.006)$ & 0.079 \\
Age, T-chol & $2.642(0.887-7.864)$ & 0.081 \\
Age, HDL-chol & $2.075(0.815-5.280)$ & 0.126 \\
Age, LDL-chol & $2.053(0.805-5.237)$ & 0.132 \\
Age, TG & $2.746(1.066-7.076)$ & $0.036^{*}$ \\
Age, AF & $2.415(0.945-6.167)$ & 0.065
\end{tabular}

Table 3. Lesional characteristics

\begin{tabular}{|c|c|c|c|c|}
\hline Multivessel disease (\%) & $53(26)$ & $15(28)$ & $38(72)$ & 0.654 \\
\hline Calcified lesions (\%) & $29(14)$ & $8(28)$ & $21(72)$ & 0.831 \\
\hline Ostial lesions (\%) & $30(15)$ & $12(40)$ & $18(60)$ & 0.058 \\
\hline CTO lesions (\%) & $12(6)$ & $4(33)$ & $8(67)$ & 0.549 \\
\hline DES use (\%) & $193(95)$ & $50(26)$ & $143(74)$ & 0.92 \\
\hline BMS use (\%) & $11(5)$ & $3(27)$ & $8(73)$ & 0.92 \\
\hline
\end{tabular}




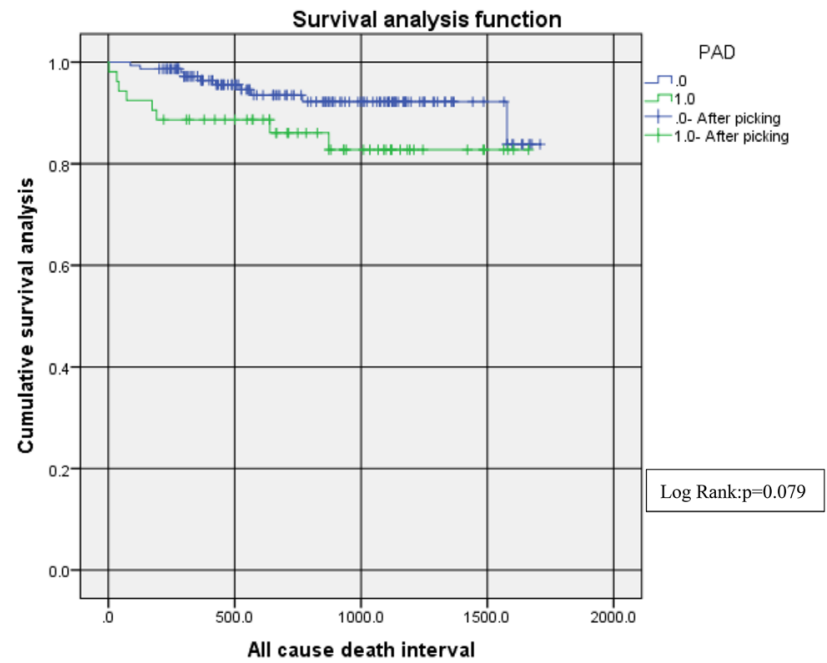

\begin{tabular}{|ll|l|l|l|l|}
\hline Follow-up (day) & 0 & 500 & 1000 & 1500 \\
\hline PAD 0 No.at risk & 151 & 99 & 51 & 12 \\
\cline { 4 - 7 } & \multirow{2}{*}{$\%$} & & 66 & 34 & 8 \\
\hline PAD 1 & No.at risk & 53 & 41 & 20 & 4 \\
\hline & & & 77 & 38 & 8 \\
\hline
\end{tabular}

Figure 1. Kaplan-Meier analysis of PAD in patients with all events. PAD patients (PAD 1) predicted all cause death events (green line). Blue line, non-PAD patients.

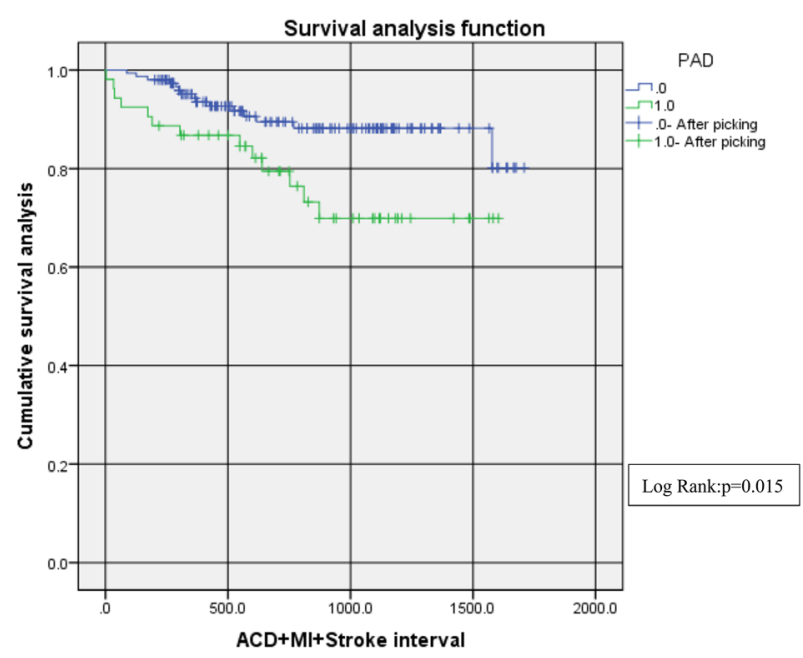

\begin{tabular}{|ll|l|l|l|l|}
\hline Follow-up (day) & 0 & 500 & 1000 & 1500 \\
\hline PAD 0 & No.at risk & 151 & 96 & 50 & 12 \\
& $\%$ & & 64 & 33 & 8 \\
\hline PAD 1 & No.at risk & 53 & 40 & 18 & 3 \\
\hline & $\%$ & & 75 & 34 & 6 \\
\hline
\end{tabular}

Figure 2. Kaplan-Meier analysis of PAD in patients with all events. PAD patients (PAD 1) predicted all-cause death+MI+Stroke events (green line). Blue line, non-PAD patients.

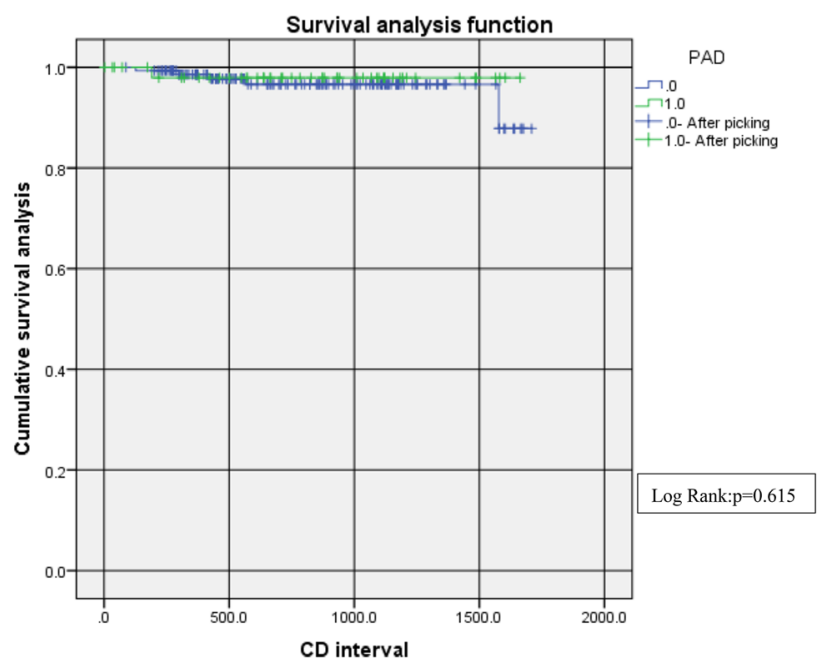

\begin{tabular}{|ll|l|l|l|l|}
\hline Follow-up (day) & 0 & 500 & 1000 & 1500 \\
\hline PAD 0 & No.at risk & 151 & 99 & 51 & 12 \\
& $\%$ & & 66 & 34 & 8 \\
\hline \multirow{2}{*}{ PAD 1 } & No.at risk & 53 & 41 & 20 & 4 \\
\hline & $\%$ & & 77 & 38 & 8 \\
\hline
\end{tabular}

Figure 3. Kaplan-Meier analysis of PAD in patients with all events. PAD patients (PAD 1) predicted cardiac death events (green line). Blue line, non-PAD patients.

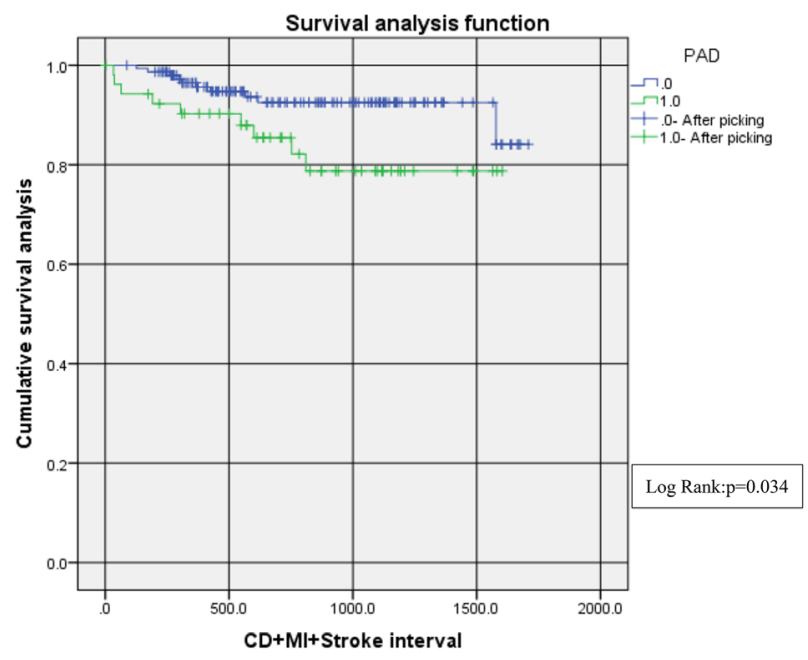

\begin{tabular}{|ll|l|l|l|l|}
\hline Follow-up (day) & 0 & 500 & 1000 & 1500 \\
\hline PAD 0 & No.at risk & 151 & 96 & 50 & 12 \\
& $\%$ & & 64 & 33 & 8 \\
\hline \multirow{2}{*}{ PAD 1 } & No.at risk & 53 & 40 & 18 & 3 \\
\hline & $\%$ & & 75 & 34 & 6 \\
\hline
\end{tabular}

Figure 4. Kaplan-Meier analysis of PAD in patients with all events. PAD patients (PAD 1) predicted cardiac death+MI+Stroke events (green line). Blue line, non-PAD patients. 
Bhatt DL, Steg PG, Ohman EM, et al. 2006. International prevalence, recognition, and treatment of cardiovascular risk factors in outpatients with atherothrombosis. Journal of the American Medical Association. 295(2):180-189.

Chiu JH, Topol EJ, Whitlow PL, et al. 2003. Peripheral vascular disease and one-year mortality following percutaneous coronary revascularization Am J Cardiol. 92:582-583.

Fowkes FG, Rudan D, Rudan I, et al. 2013. Comparison of global estimates of prevalence and risk factors for peripheral artery disease in 2000 and 2010: a systematic review and analysis. Lancet. 382(9901):1329 1340.

Global, regional, and national incidence, prevalence, and years lived with disability for 328 diseases and injuries for 195 countries, 1990-2016: a systematic analysis for the Global Burden of Disease Study 2016[J]. 2017. Lancet. 390(10100):1211-1259.

McKenna M, Wolfson S, Kuller L. 1991. The ratio of ankle and arm arterial pressure as an independent predictor of mortality. Atherosclerosis. 87: 119-128.

Morris DR, Rodriguez AJ, Golledge J. 2014. Association of Lower Extremity Performance with Cardiovascular and All-Cause Mortality in Patients with Peripheral Artery Disease: A Systematic Review and Metaanalysis. J Am Heart Assoc. 3: e001-105.

Nikolsky E, Mehran R, Mintz GS, et al. 2004. Impact of symptomatic peripheral arterial disease on 1-year mortality in patients undergoing percutaneous coronary interventions.J Endovasc Ther. 11:60-70.

Nishijima A, Yamamoto N, Yoshida R, Hozawa K, Yanagibayashi S, et al. 2017. Coronary Artery Disease in Patients with Critical Limb Ischemia Undergoing Major Amputation or Not. Plast Reconstr Surg Glob Open. 5: e1377.

Norgren L, Hiatt WR, Dormandy JA, Nehler MR, Harris KA, Fowkes

FG, et al. 2007. TA SC II Working Group. Inter-Society Consensus for the Management of Peripheral Arterial Disease (TASC II). Eur J Vasc
Endovasc

Surg. 33(Suppl 1): S1-75.

O'Connor GT, Malenka DJ, Quinton H, et al. for the Northern New England Cardiovascular Disease Study Group. 1999. Multivariate prediction of in-hospital mortality after percutaneous coronary interventions in 1994-1996. J Am Coll Cardiol. 34:681-91.

Olin JW, Allie DE, Belkin M, et al. 2010. CCF/AHA/ACR/SCAI/SIR/ SVM/SVN/SVS 2010 performance measures for adults with peripheral artery disease. Vasc Med. 15(6):481-512.

Olinic DM, Spinu M, Olinic M, Homorodean C, Tataru DA, Liew A, Schernthaner GH, Stanek A, Fowkes G, Catalano M. 2018. Epidemiology of peripheral artery disease in Europe: VAS Educational Paper. Int Angiol. 37(4):327-334.

Roth GA, Johnson C, Abajobir A, et al. 2017. Global, Regional, and National Burden of Cardiovascular Diseases for 10 Causes, 1990 to 2015[J]. Journal of the American College of Cardiology. 70(1):1-25.

Ryu HM, Kim JS, Ko YG, Hong MK, Jang Y, et al. 2012. Clinical outcomes of infrapopliteal angioplasty in patients with critical limb ischemia. Korean Circ J. 42: 259-265.

Saw J, Bhatt DL, Moliterno DJ, Brener SJ, Steinhubl SR, Lincoff AM, Tcheng JE, Harrington RA, Simoons M, Hu T, Sheikh MA, Kereiakes DJ, Topol EJ. 2006. The influence of peripheral arterial disease on outcomes: a pooled analysis of mortality in eight large randomized percutaneous coronary intervention trials. J Am Coll Cardiol. 48(8):1567-72.

Singh M, Lennon R, Darbar D, et al. 2004. Effect of peripheral arterial disease in patients undergoing percutaneous coronary intervention with intracoronary stents. Mayo Clin Proc. 79:1113-1118.

Suzuki S, Hashizume N, Kanzaki Y, Maruyama T, Kozuka A, Yahikozawa K. 2019. Prognostic significance of serum albumin in patients with stable coronary artery disease treated by percutaneous coronary intervention. PLoS One. 14(7): e0219044. 